\title{
Ultrasonic-based filter aided sample preparation as the universal method to sample preparation in proteomics.
}

Luís B. Carvalho $\uparrow$, , José-Luis Capelo-Martínez $\uparrow, \ddagger$, Carlos Lodeiro $\uparrow, \star$, Jacek R. Wiśniewski $\S$, Hugo M. Santos $\uparrow, \ldots, \|, *$

$\uparrow$ BIOSCOPE Research Group, LAQV-REQUIMTE, Department of Chemistry, Faculty of Science and Technology, Universidade NOVA de Lisboa, Campus de Caparica, Caparica 2829-516, Portugal.

* PROTEOMASS Scientific Society, Madan Parque, Rua dos Inventores, Caparica 2825-182, Portugal.

$\S$ Biochemical Proteomics Group, Department of Proteomics and Signal Transduction, Max-Planck-Institute of Biochemistry, Am Klopferspitz 18, Martinsried D-82152, Germany.

"I Department of Pathology, University of Pittsburgh Medical Center, Pittsburgh, Pennsylvania 15213 United States.

*Corresponding Author: Tel.: +351 934432 320. E-mail: hmsantos@fct.unl.pt

\section{Contents of the Supporting Information}

Reagents

Apparatus

SI 1 - E. coli proteome.

SI 2 - Mouse liver and brain proteomes.

SI 3 - Total protein and peptide quantification

SI 4 - Nano-LC-MS/MS

Figure SI 5 - Optical microscopy images (40x) from the FASP membranes using the conditions (A)

Control, (B) overnight digestion and (C) ultrasonic digestion. The images on top are the original images from the microscope, and in the images at the bottom color has been adjusted.

Figure SI 6 - Hierarchal clustering of the E. coli proteome processed with US-FASP and ON-FASP. 1053 proteins identified in each method (US-FASP and ON-FASP) with a filter of $70 \%$ presence in at least one group. Protein label free intensities normalized by Z-score were plotted in a hierarchal clustering using average as linkage.

Figure SI 7 - Custom-built wood plate vivacon support floater. 


\section{Reagents}

All reagents used were HPLC grade or electrophoresis grade. Bovine serum albumin (BSA), $\alpha$-lactalbumin from bovine milk, $\beta$-lactoglobulin from bovine milk, $\alpha$-casein from bovine milk, iodoacetamide (IAA), urea, phosphate buffered saline (PBS), formic acid (FA), tris base and Bradford reagent were purchased from Sigma-Aldrich (Basel, Switzerland). Ammonium bicarbonate (AmBic) and hydrochloric acid solution 37\% $(\mathrm{HCl})$ were purchased from Fluka (Basel, Switzerland). Sodium dodecyl sulfate (SDS), acetonitrile (ACN) and boric acid were purchased from Panreac (Barcelona, Spain). 1,4-Dithio-DL-threitol (DTT) was purchased from Alfa Aesar (Haverhill, Massachusetts, USA). Pierce ${ }^{\mathrm{TM}}$ trypsin protease MS grade, trifluoroacetic acid (TFA) and pierce ${ }^{\mathrm{TM}}$ quantitative colorimetric peptide assay were purchased from Thermo Fisher Scientific (Waltham, Massachusetts, USA). 


\section{Apparatus}

Ultrasonic processor Branson SLPe digital sonifier with a probe of $1 \mathrm{~mm}$ was utilized for tissue homogenization. The membrane vivacon 500 10,000 and 30,000 MWCO Hydrosart from Sartorius (Goettingen, Germany) and prism ${ }^{\mathrm{TM}}$ refrigerated microcentrifuge from Labnet (New Jersey, USA) were used in the standard FASP and ultrasound FASP methodology. A mini incubator from Labnet (New Jersey, USA) was used for protein reduction and digestion in the standard FASP method. Microplate Horn Assembly equipped with a water recirculation system and operating with the Q700 system (20 kHz) from QSonica (Newtown, CT, USA) was used to accelerate the steps of reduction, alkylation and digestion in the ultrasound FASP. A custombuilt wood plate vivacon support $(10.5 \mathrm{~cm} \times 9.9 \mathrm{~cm} \times 9.9 \mathrm{~cm}$ ) was used in the ultrasound FASP steps. A vacuum concentrator centrifuge model UNIVAPO 150 ECH Speed Vac and a vacuum pump model UNIJET II (Munich, Germany) were used for sample drying. Vortex models ELMI CM70M-09 from SkyLine (Southern California, USA) and VX-200 Vortex Mixer from Labnet (New Jersey, USA) were used in sample mix. CLARIOstar® High Performance Monochromator Multimode from BMG LABTECH (Germany) was used for Bradford and total peptide assays. Acquisition of mass spectrometry data was done using an UHR-QqTOF IMPACT HD from Bruker Daltonics (Bremen, Germany) Chromatographic separation of peptides were carried out using an Ultimate 3000 nLC nano-system equipped with a trap-column Acclaim PepMap100, $5 \mu \mathrm{m}, 100$ A, $300 \mu \mathrm{m}$ i.d. $\times 5$ mm (Thermo Fisher Scientific) and an analytical column Acclaim ${ }^{\mathrm{TM}}$ PepMap $^{\mathrm{TM}} 100 \mathrm{C} 18,2 \mu \mathrm{m}, 0.075 \mathrm{~mm}$ i.d x 150mm (Thermo Fisher Scientific). 


\section{SI 1 - E. coli proteome.}

E. coli samples were prepared using the ReadyPrepTM E. coli lysate sample from BioRad, following manufactures' instructions with minor modifications. Briefly, $500 \mu \mathrm{L}$ of $3 \mathrm{M}$ Urea $25 \mathrm{mM}$ ammonium bicarbonate (AmBic) buffer solution were added to the $E$. coli extracts and incubated for five min at room temperature, following by five min sonication using an ultrasonic bath at $100 \%$ ultrasonic amplitude, UA, $35 \mathrm{kHz}$ ultrasonic frequency, UF, to ensure that the dried E. coli protein extracts were solubilized. Next, several aliquots of $30 \mu \mathrm{L}$ were prepared, followed by storage at $-60{ }^{\circ} \mathrm{C}$ until further use. Furthermore, in some experiments to access the different methods reproducibility, five different known concentrations of bovine serum albumin (BSA), $\beta$-lactoglobulin, $\alpha$-casein, and $\alpha$-lactalbumin were spiked to the $E$. coli proteome. 


\section{SI 2 - Mouse liver and brain proteomes.}

Fresh frozen mouse liver and brain were obtained from Patricell Limited. Tissue samples were weight and washed with cold $0.1 \mathrm{M}$ phosphate buffer solution (PBS) $4{ }^{\circ} \mathrm{C}$ to remove traces of blood. Next, the tissues were homogenized in a lysis buffer 0.1 M Tris-HCl, pH 7.8, $0.05 \mathrm{M}$ DTT 2\% SDS (w/v) using a Potter-Elvehjem tissue grinder. The ratio of tissue mass to lysis buffer was 1:10 $(\mathrm{g} / \mathrm{mL})$. The homogenates were then sonicated for 2 min on ice $(30 \mathrm{~s}$ on $10 \mathrm{~s}$ off ultrasonic time, UT, 50\% UA, $40 \mathrm{kHz}$ UF, $1 \mathrm{~mm}$ diameter probe) using a Branson SLPe digital sonifier, followed by incubation at $95{ }^{\circ} \mathrm{C}$ in a water bath for five min. After cooling to room temperature, tissue lysates were clarified by centrifugation at $16000 \mathrm{~g}$ for $10 \mathrm{~min}$. As final step the supernatant was transferred into aliquots of $1 \mathrm{~mL}$ and stored at $-60{ }^{\circ} \mathrm{C}$ until further use. 


\section{SI 3 - Total protein and peptide quantification}

The total protein present in the E. coli, mouse brain and mouse liver were quantified by Bradford protein assay. After digestion, peptides were quantified using the Thermo Fisher Scientific pierce ${ }^{\mathrm{TM}}$ quantitative colorimetric peptide assay following manufacturer's instructions. 


\section{SI 4 - Nano-LC-MS/MS}

LC-MS/MS analysis was carried out using a Ultimate 3000 nano LC system coupled to an Impact HD (Bruker Daltonics) with a CaptiveSpray nanoBooster using acetonitrile as dopant Peptides were resuspended in $100 \mu \mathrm{L}$ of $3 \%(\mathrm{v} / \mathrm{v})$ acetonitrile containing $0.1 \%(\mathrm{v} / \mathrm{v})$ aqueous formic acid (FA). Then, samples were homogenized by $5 \mathrm{~min}$ on vortex followed by $10 \mathrm{~min}$ on an ultrasonic bath at $100 \%$ UA, $35 \mathrm{kHz}$ UF. Afterwards, samples were quantified using a pierce $^{\mathrm{TM}}$ quantitative colorimetric peptide assay. Following the previous step, $5 \mu \mathrm{L}$ containing $750 \pm 5 \mathrm{ng}$ of peptides were loaded onto a trap column (Acclaim PepMap100, $5 \mu \mathrm{m}, 100 \AA$, $300 \mu \mathrm{m}$ i.d. $\times 5 \mathrm{~mm}$ ) and desalted for $5 \mathrm{~min}$ from $3 \%$ to $5 \% \mathrm{~B}$ (B: $90 \%$ acetonitrile $0.08 \% \mathrm{FA}$ ) at a flow rate of $15 \mu \mathrm{L}$.min-1. Then the peptides were separated using an analytical column $\left(\right.$ Acclaim $^{\text {TM }}$ PepMap ${ }^{\text {TM }} 100 \mathrm{C} 18,2 \mu \mathrm{m}, 0.075 \mathrm{~mm}$ i.d x $150 \mathrm{~mm}$ ) with a linear gradient at 300 nL.min-1 (mobile phase A: aqueous FA 0.1\% (v/v); mobile phase B 90\% (v/v) acetonitrile and $0.08 \%(\mathrm{v} / \mathrm{v}) \mathrm{FA}$ ) $5-90 \mathrm{~min}$ from $5 \%$ to $35 \%$ of mobile phase B, 90-100min linear gradient from $35 \%$ to $95 \%$ of mobile phase B, 100-110 95\% B. Chromatographic separation was carried out at $35{ }^{\circ} \mathrm{C}$. MS acquisition was set to cycles of MS (2 Hz), followed by MS/MS (8-32 Hz), cycle time $3.0 \mathrm{~s}$, with active exclusion (precursors were excluded from precursor selection for 0.5 min after acquisition of $1 \mathrm{MS} / \mathrm{MS}$ spectrum, intensity threshold for fragmentation of 2500 counts). Together with active exclusion set to 1 , reconsider precursor if the intensity of a precursor increases by a factor of 3 , this mass was taken from temporarily exclusion list and fragmented again, ensuring that fragment spectra were taken near to the peak maximum. All spectra were acquired in the range $150-2200 \mathrm{~m} / \mathrm{z}$. 

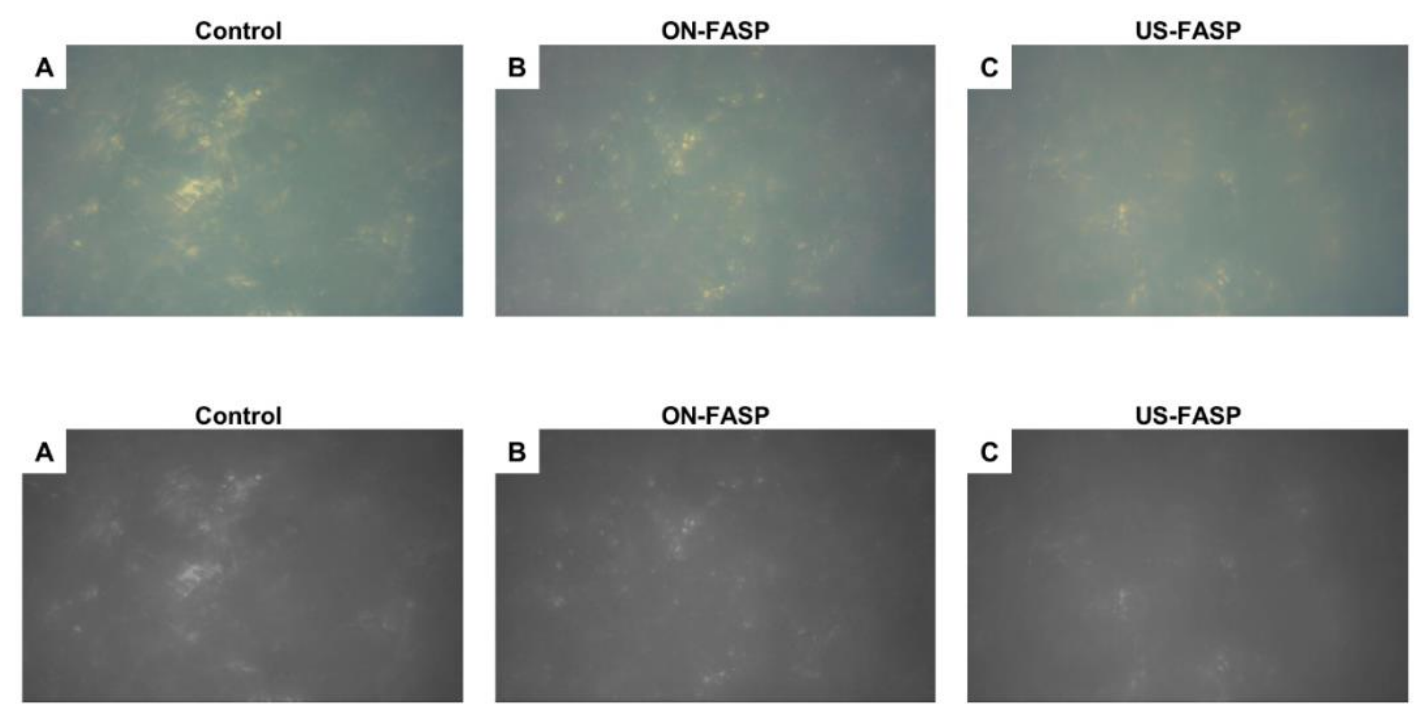

Figure SI 5 - Optical microscopy images (40x) from the FASP membranes using the conditions (A) Control, (B) overnight digestion and (C) ultrasonic digestion. The images on top are the original images from the microscope, and in the images at the bottom color has been adjusted. 


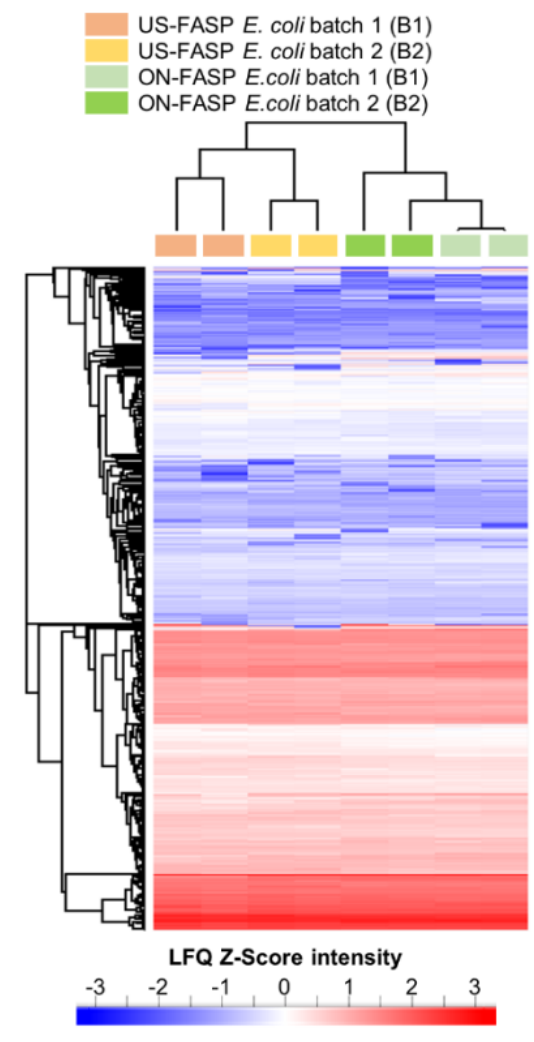

Figure SI 6 - Hierarchal clustering of the $E$. coli proteome processed with US-FASP and ON-FASP. 1053 proteins identified in each method (US-FASP and ON-FASP) with a filter of $70 \%$ presence in at least one group. Protein label free intensities normalized by Z-score were plotted in a hierarchal clustering using average as linkage. 


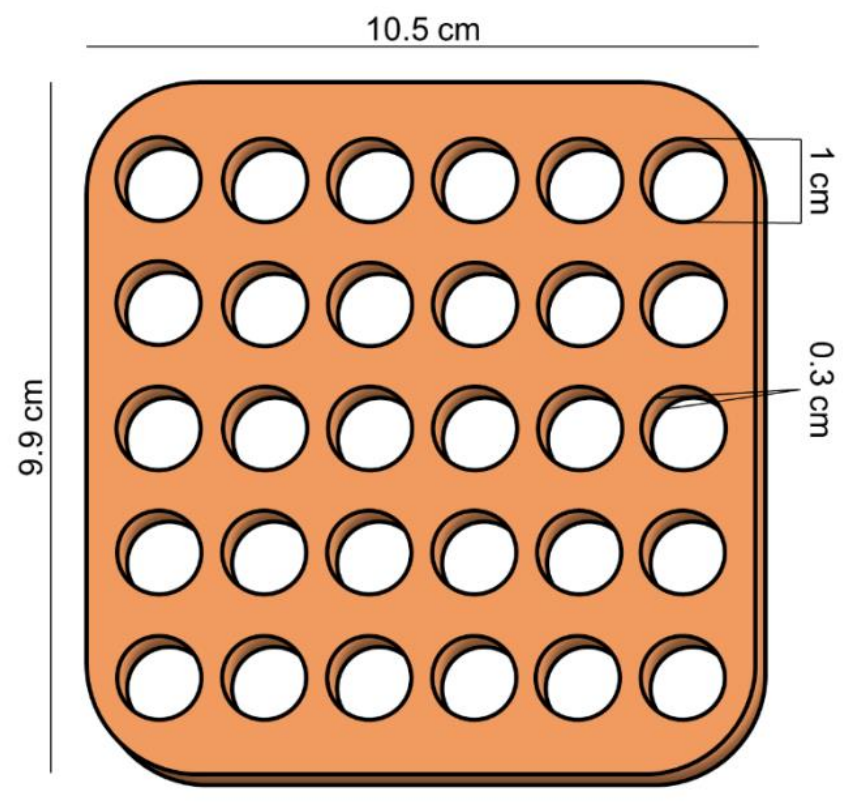

Figure SI 7 - Custom-built wood plate vivacon support floater. 